East Tennessee State University

Digital Commons@ East Tennessee State University

ETSU Faculty Works

Faculty Works

3-6-2019

\title{
Coordinated UV and X-Ray Spectroscopic Observations of the O-type Giant $\xi$ Per: The Connection between X-Rays and Large-scale Wind Structure
}

Derek Massa

Space Science Institute

Lidi Oskinova

University of Potsdam

Raman Prinja

East Tennessee State University

Richard Ignace

East Tennessee State University, ignace@etsu.edu

Follow this and additional works at: https://dc.etsu.edu/etsu-works

\section{Citation Information}

Massa, Derek; Oskinova, Lidi; Prinja, Raman; and Ignace, Richard. 2019. Coordinated UV and X-Ray Spectroscopic Observations of the O-type Giant $\xi$ Per: The Connection between X-Rays and Large-scale Wind Structure. Astrophysical Journal. Vol.873(1).

https://iopscience.iop.org/article/10.3847/1538-4357/ab0283/meta ISSN: 1538-4357 


\section{Coordinated UV and X-Ray Spectroscopic Observations of the O-type Giant $\xi$ Per: The Connection between X-Rays and Large-scale Wind Structure}

\section{Copyright Statement}

(C) 2019. The American Astronomical Society. Reproduced by permission of the AAS. 


\title{
Coordinated UV and X-Ray Spectroscopic Observations of the O-type Giant $\xi$ Per: The Connection between X-Rays and Large-scale Wind Structure ${ }^{* \dagger}$
}

\author{
Derck Massa $^{1}$ (1), Lida Oskinova ${ }^{2,3}$, Raman Prinja ${ }^{4}$, and Richard Ignace ${ }^{5}$ (1) \\ ${ }^{1}$ Space Science Institute, 4750 Walnut Street, Suite 205, Boulder, CO 80301, USA; dmassa@ spacescience.org \\ ${ }^{2}$ Institute for Physics and Astronomy, University of Potsdam, Karl-Liebknecht-Str. 24/25, D-14476 Potsdam, Germany \\ ${ }^{3}$ Kazan Federal University, Kremlevskaya Str. 18, 420008, Kazan, Russia \\ ${ }^{4}$ Department of Physics \& Astronomy, University College London, Gower Street, London WC1E 6BT, UK \\ 5 East Tennessee State University, Department of Physics \& Astronomy, Johnson City, TN 37614 USA \\ Received 2018 August 22; revised 2019 January 11; accepted 2019 January 27; published 2019 March 6
}

\begin{abstract}
We present new, contemporaneous Hubble Space Telescope STIS and XMM-Newton observations of the O7 III(n) ((f)) star $\xi$ Per. We supplement the new data with archival IUE spectra, to analyze the variability of the wind lines and X-ray flux of $\xi$ Per. The variable wind of this star is known to have a 2.086-day periodicity. We use a simple, heuristic spot model that fits the low-velocity (near-surface) IUE wind line variability very well, to demonstrate that the low-velocity absorption in the new STIS spectra of N IV $\lambda 1718$ and Si IV $\lambda 1402$ vary with the same 2.086-day period. It is remarkable that the period and amplitude of the STIS data agree with those of the IUE spectra obtained $22 \mathrm{yr}$ earlier. We also show that the time variability of the new XMM-Newton fluxes is also consistent with the 2.086-day period. Thus, our new, multiwavelength coordinated observations demonstrate that the mechanism that causes the UV wind line variability is also responsible for a significant fraction of the $\mathrm{X}$-rays in single $\mathrm{O}$ stars. The sequence of events for the multiwavelength light-curve minima is Si IV $\lambda 1402, \mathrm{~N}$ IV $\lambda 1718$, and X-ray flux, each separated by a phase of about 0.06 relative to the 2.086-day period. Analysis of the X-ray fluxes shows that they become softer as they weaken. This is contrary to expectations if the variability is caused by periodic excess absorption. Furthermore, the high-resolution X-ray spectra suggest that the individual emission lines at maximum are more strongly blueshifted. If we interpret the low-velocity wind line light curves in terms of our model, it implies that there are two bright regions, i.e., regions with less absorption, separated by $180^{\circ}$, on the surface of the star. We note that the presence and persistence of two spots separated by $180^{\circ}$ suggest that a weak dipole magnetic field is responsible for the variability of the UV wind line absorption and X-ray flux in $\xi$ Per.
\end{abstract}

Key words: stars: activity - stars: early-type - stars: winds, outflows - ultraviolet: stars - X-rays: stars

\section{Introduction}

$\mathrm{O}$ star $\left(M \gtrsim 20 M_{\odot}\right)$ winds input significant mechanical energy into the interstellar medium and affect the evolution of their host clusters and galaxies. Mass loss by winds also determines the ultimate fate of massive stars and the nature of their remnants. Consequently, reliable measurements of massloss rates due to stellar winds are essential for all of these subjects. Stellar winds are driven by radiative pressure on metal lines (Castor et al. 1975). Because the optical spectra of O stars are dominated by photospheric lines or recombination lines (which only sample the very base of the wind), much of wind research has concentrated on UV resonance lines, which are formed throughout the wind.

Over the years, it has become apparent that radiatively driven winds are far more complex than the homogeneous, spherically symmetric flows envisioned by Castor et al. (1975). Instead, they have been shown to contain optically thick structures that can be quite small or very large. Until we unravel the details of these flows, we cannot hope to reliably translate observational diagnostics into physical quantities such

\footnotetext{
* Based on observations made with the NASA/ESA Hubble Space Telescope, obtained at the Space Telescope Science Institute, which is operated by the Association of Universities for Research in Astronomy, Inc., under NASA contract NAS 5-26555. These observations are associated with program \#13760.

$\dagger$ Based on observations obtained with XMM-Newton, an ESA science mission with instruments and contributions directly funded by ESA Member States and NASA.
}

as mass-loss rates. To progress, we need a firm grasp on the underlying physical mechanisms that determine the wind structures. The state of affairs can be seen in the literature, where the values of observationally derived mass-loss rates have swung back and forth by factors of 10 or more (Massa et al. 2003; Fullerton et al. 2006; Puls et al. 2006; Oskinova et al. 2007; Sundqvist et al. 2011; Šurlan et al. 2012).

The presence of large structures was revealed in time series of unsaturated UV wind lines. Dynamic spectra of these lines display discrete absorption components (DACs) that indicate large, coherent structures propagating through the winds (e.g., Kaper et al. 1999; Prinja et al. 2002). Similar features are observed in LMC and SMC O stars (Massa et al. 2000) and in central stars of planetary nebula (Prinja et al. 2012), suggesting that they are a universal property of radiatively driven flows. Furthermore, Prinja \& Massa (2010) demonstrated that the wind lines in many OB stars have anomalous doublet ratios, indicating that the winds have optically thick structures embedded within them. Cranmer \& Owocki (1996) showed how the spiral structures produced by corotating interaction regions (CIRs; e.g., Mullan 1984) can explain UV wind line variability by introducing bright spots near the equator. The CIR model was applied to $\xi$ Per, O7.5 III(n)((f)) (de Jong et al. 2001), and HD 64760, B0.5Ib (Fullerton et al. 1997; Lobel \& Blomme 2008), and its signature also appears in the dynamic spectra of most long-duration time series (e.g., Prinja et al. 2002; Massa \& Prinja 2015). The CIR model predicts spiral structures with density enhancements of $\sim 2$, which, together 
with velocity plateaus, can increase Sobolev optical depths by factors of 10 to 100 . While CIRs may have little effect on the mass-loss rate, they can strongly affect the observational diagnostics used to determine it.

Another indication of wind structure is the universal presence of X-ray emission from $\mathrm{O}$ stars. Further, there is growing evidence that X-ray fluxes vary on timescales of days (Oskinova et al. 2001; Nazé et al. 2013, 2018; Massa et al. 2014; Nichols et al. 2015; Rauw et al. 2015).

As yet, the continuous X-ray monitoring, needed to determine whether this variability is periodic, is lacking. The $\mathrm{X}$-ray spectra of OB stars imply that the X-rays are produced throughout the wind, arising from the mechanical energy of impacts between different wind components. It is widely accepted that the X-rays arise from the line-deshadowing instability (LDI; e.g., Lucy \& White 1980; Owocki et al. 1988; Feldmeier et al. 1997) In LDI models, a component of the wind flow fragments into randomly distributed structures that interact with each other or the ambient wind to produce X-rays. Some LDI predictions agree with observations. For example, X-ray emission line profiles (e.g., Oskinova et al. 2006; Hervé et al. 2013; Cohen et al. 2014) imply that the hot plasma moves with the same velocity as the cool wind and that the X-rays are attenuated by the cool gas component, as expected. The doublet anomaly can also be interpreted in terms of LDI fragments (Sundqvist et al. 2011; Šurlan et al. 2012). However, other predictions are at odds with the observations. The model predicts strong short-timescale stochastic X-ray variability (Feldmeier et al. 1997), while none is observed (Nazé et al. 2013). It also predicts that the strongest shocks (hottest plasma) should originate well out in wind, while observations indicate that the hottest plasma is very close to the photosphere (Waldron \& Cassinelli 2007). Further, the LDI model cannot explain X-ray variability on a timescale of days. From a theoretical perspective, it is not yet clear whether LDI clumping and large-scale CIR structures can coexist (Sundqvist et al. 2018) or whether LDI models can produce the observed levels of X-ray fluxes when three-dimensional effects are included (Steinberg \& Metzger 2018).

CIRs provide an obvious, but untested, means for producing $\mathrm{X}$-ray variability, either by creating X-rays at their interfaces with the freely flowing wind (Mullan 1984; Ignace et al. 2013) or by modulating the ambient X-ray flux by their density enhancements. Two observational results that could enable us to make progress are whether the X-ray emission varies periodically and whether it is related to the UV wind line variability. If these are the case, it would indicate a CIR-X-ray connection that could help constrain future modeling of how the CIRs and X-ray sources interact and their relative locations. These tests can be performed by selecting a normal star that is bright in X-rays and has a well-documented DAC period and observing in both the X-rays and UV simultaneously for more than one period. These observations would determine whether the UV variability and X-ray variability are related, and they are the object of the current study.

This paper is organized as follows: Section 2 gives some relevant properties of $\xi$ Per, Section 3 presents the Hubble Space Telescope (HST) and XMM-Newton observations, Section 4 describes our analysis, and Section 5 summarizes and discusses the results.

\section{2. $\xi$ Per-a Rapidly Rotating O-type Giant}

$\xi$ Per is a normal, single $\mathrm{O} 7 \mathrm{III}(\mathrm{n})((\mathrm{f}))$ star with a large, but not abnormal, rotational velocity, $v \sin i=204 \mathrm{~km} \mathrm{~s}^{-1}$, and a wind terminal velocity $v_{\infty}=2450 \mathrm{~km} \mathrm{~s}^{-1}$ (de Jong et al. 2001), which is typical for its spectral type. A detailed analysis of the STIS spectra will be presented in a forthcoming study by W.-R. Hamann et al. (2019, in preparation).

$\xi$ Per has long been a favorite star for studying wind variability. It is bright, and several of its UV wind lines are well developed but unsaturated, making them ideal for examining variability. Dynamic spectra of its UV wind lines show distinct, isolated, large-amplitude, repeating DACs with a well-defined 2.086-day period (de Jong et al. 2001) that persists for more than 10 days. They assume that this period is roughly half of the actual rotation period and that two distinct structures, separated by $180^{\circ}$, are present in the wind. Their assumption is bolstered by Ramiaramanantsoa et al. (2014), who obtained 4 weeks of contiguous, high-precision visual photometry of $\xi$ Per. They demonstrated that the observed variability was consistent with localized magnetic spots on a star whose rotation period is 4.18 days. Nevertheless, it is possible that 2.086 days could be the actual stellar rotation period (see the Appendix).

In addition to UV wind line variability, Massa et al. (2014) observed $\xi$ Per for $162 \mathrm{ks}$ (1.88 days) with the Chandra HETGS. Their results showed that the X-ray flux clearly varied. However, the time baseline of their observations was not sufficient to determine whether the variation was periodic, as expected if the X-ray variability is associated with the same wind structures that create the DACs.

\section{The Observations}

This section describes the new observations. We begin with the motivation for the time sampling employed and then discuss the reductions of the HST STIS and XMM-Newton data used in the analysis.

\subsection{Time Sampling}

Our joint HST/XMM-Newton program requested time sampling based on the 2.086-day period. We proposed to observe $\xi$ Per 11 times with HST STIS over a time interval of roughly $100 \mathrm{hr}(\sim 5$ days) to capture a repeat of the DAC activity in the UV wind lines. The $H S T$ observations were to be supplemented by $107 \mathrm{ks}$ XMM-Newton observations distributed over $245 \mathrm{hr}$ and centered on the HST observations. These were to be obtained at 10 and $48 \mathrm{hr}$ intervals, as allowed by the $X M M$-Newton orbit, and sample the 2.086-day period over five periods.

While the HST STIS observations were performed as requested, XMM-Newton scheduling constraints turned out to be more severe than anticipated. Instead of a more uniform sampling over the interval, we were allocated two long (82.9 and $82.4 \mathrm{ks}$ ) exposures separated by 22.65 days. Although we were actually given more observing time than requested, the temporal spacing of the observations was far from optimal. The final time sampling of the observations is depicted in Figure 1. It shows that the STIS observations span 2.4 periods and that each XMM-Newton observation spans roughly 0.46 periods and the second begins 10.52 periods after the first. Consequently, although the XMM-Newton observations sample the 2.086-day 


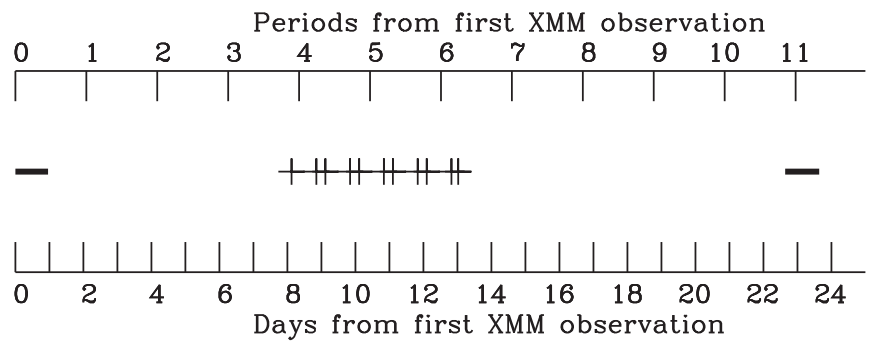

Figure 1. Observations displayed in time, with the time of the first XMMNewton observation set to zero. The lower scale is in days, and the upper scale gives the number of 2.086-day periods. The times covered by the XMMNewton observations are shown as the solid bars and the STIS observations as crosses.

period very well, none of them sample the same phase more than once.

\subsection{The STIS Data}

The STIS observations used the $R=45,800$ E140M grating with the $0.2 \times 0.05 \mathrm{ND}$ aperture, which includes a neutral density filter, necessitated because $\xi$ Per is so bright. The spectra include the important Si IV $\lambda 1400$ resonance doublet and the N IV $\lambda 1718$ excited state line.

Creation of the Si IV $\lambda 1402$ fluxes was relatively straightforward. The only complication was that the individual spectra had to be rectified to the band $1.0 \leqslant v / v_{\infty} \leqslant 1.5$. This was necessary because the overall flux levels of E140M spectra obtained through the small $0.2 \mathrm{X} 0.05 \mathrm{ND}$ aperture can vary owing to differences in the positioning of the object in the aperture and variations in the image size resulting from telescope "breathing" due to orbital thermal variations (Proffitt et al. 2017).

The extraction of the N IV data was more complex. Unfortunately, the standard STScI pipeline processing of objects observed recently by the STIS E140M omits the last echelle order, which contains $1718 \AA$ (although earlier spectra include it). Consequently, we had to extract this order by hand.

Figure 2 shows the portion of the far-UV MAMA detector that includes the order containing N IV $\lambda 1718$ (the upper stripe). Spectra for this order were extracted using a simple box car. Specifically, we first determine the mean counts for $1000 \leqslant y \leqslant 1015$ as a function of $x$, termed the gross spectrum. Similarly, a background spectrum was determined from the means over $985 \leqslant y \leqslant 1000$. The background was then subtracted from the gross to give a set of un-fluxcalibrated net spectra. Figure 3 gives an example of the gross, background, and net spectra.

The wavelength alignment of the net spectra appears quite good (the heliocentric velocities varied by less than $0.2 \mathrm{~km} \mathrm{~s}^{-1}$ ). The wavelength scale was determined by using the STIS dispersion relation applied to spectra that include the order containing $1718 \AA$. This is $\lambda=0.053619 x+b \AA$, where $b=1710.75 \pm 0.1 \AA$, depending on the location of the star and the velocity of the telescope. An uncertainty of $\pm 0.1 \AA$ is not important for our purposes. The wavelength scale is only used to determine the portion of the spectrum to be averaged for studying N IV $\lambda 1718$ flux variations. Since an uncertainty of $0.1 \AA$ only amounts to $17 \mathrm{~km} \mathrm{~s}^{-1}$, or $\Delta v / v_{\infty}<1 \%$, it has an insignificant effect on the mean fluxes.

As mentioned above, the individual spectra had to be rectified to the same mean. In this case we used the band

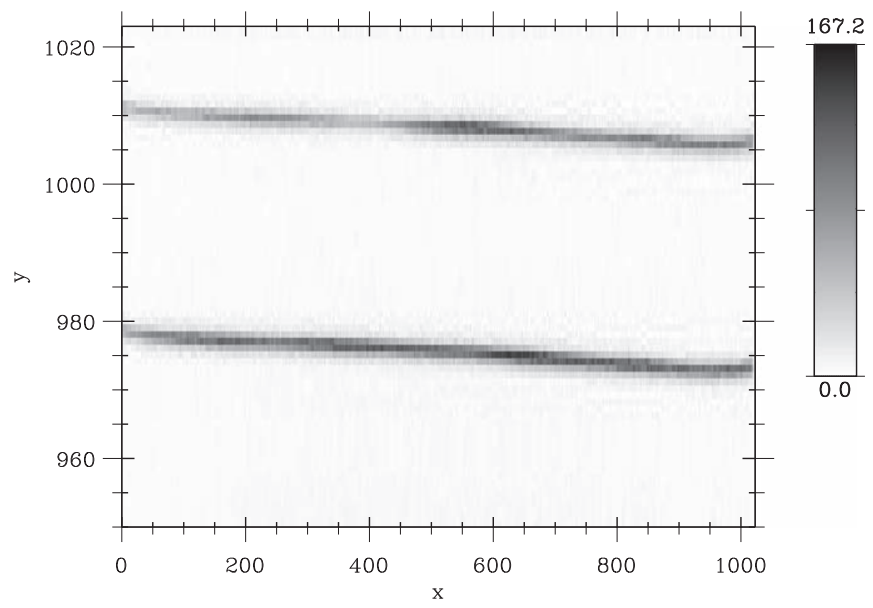

Figure 2. Image of the portion of the STIS far-UV MAMA detector containing $\mathrm{N}$ IV $\lambda 1718$, the line appearing in the top order, centered near $x=400$.

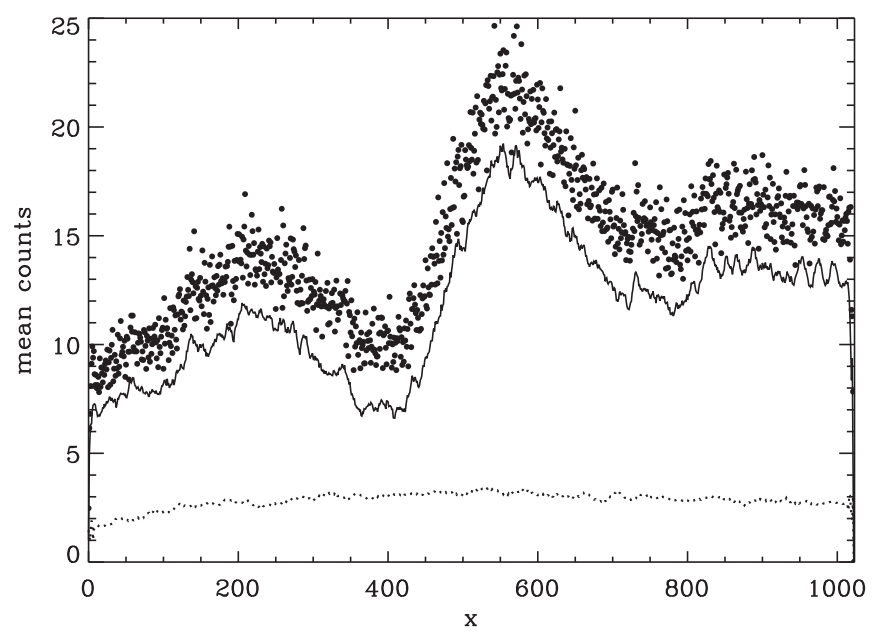

Figure 3. Raw spectrum (points), background smoothed by 16 points (dotted curve), and net spectrum (raw minus background) smoothed by eight points (solid curve).

$800 \leqslant x \leqslant 1000$, which corresponds to $1753.7 \leqslant \lambda \leqslant 1764.4$, which is well away from expected wind activity. Since subsequent measurements are normalized by their means, absolute flux calibration is not needed.

\subsection{The XMM-Newton Data}

XMM-Newton observed $\xi$ Per on two separate visits, first for an 82.9 ks exposure on 2016 February 6 (ObsID 0770990101), and again for an $82.4 \mathrm{ks}$ exposure on 2016 February 28 (ObsID 0770990201) (see Figure 1). The three XMM-Newton X-ray telescopes illuminate five instruments that operate simultaneously and independently: two reflection grating spectrometers (RGS1 and RGS2; den Herder et al. 2001), with a spectral resolution of $\sim 0.07 \AA$ and wavelength coverage of $5 \AA \lesssim \lambda \lesssim 38 \AA$, and three focal plane instruments, forming the European Photon Imaging camera (EPIC). The EPIC camera consists of the MOS1 and MOS2 (Metal-Oxide Semiconductor) and pn (pn-CCDs) detectors. The EPIC instruments cover $1.2 \AA \lesssim \lambda \lesssim 60 \AA$, with a spectral resolution of $E / \Delta E \approx 20-50$ (Strüder et al. 2001; Turner et al. 2001). During each observation, all three EPIC cameras were operated in the standard, full-frame mode with a thick UV filter, and simultaneous RGS1 and RGS2 spectra were 


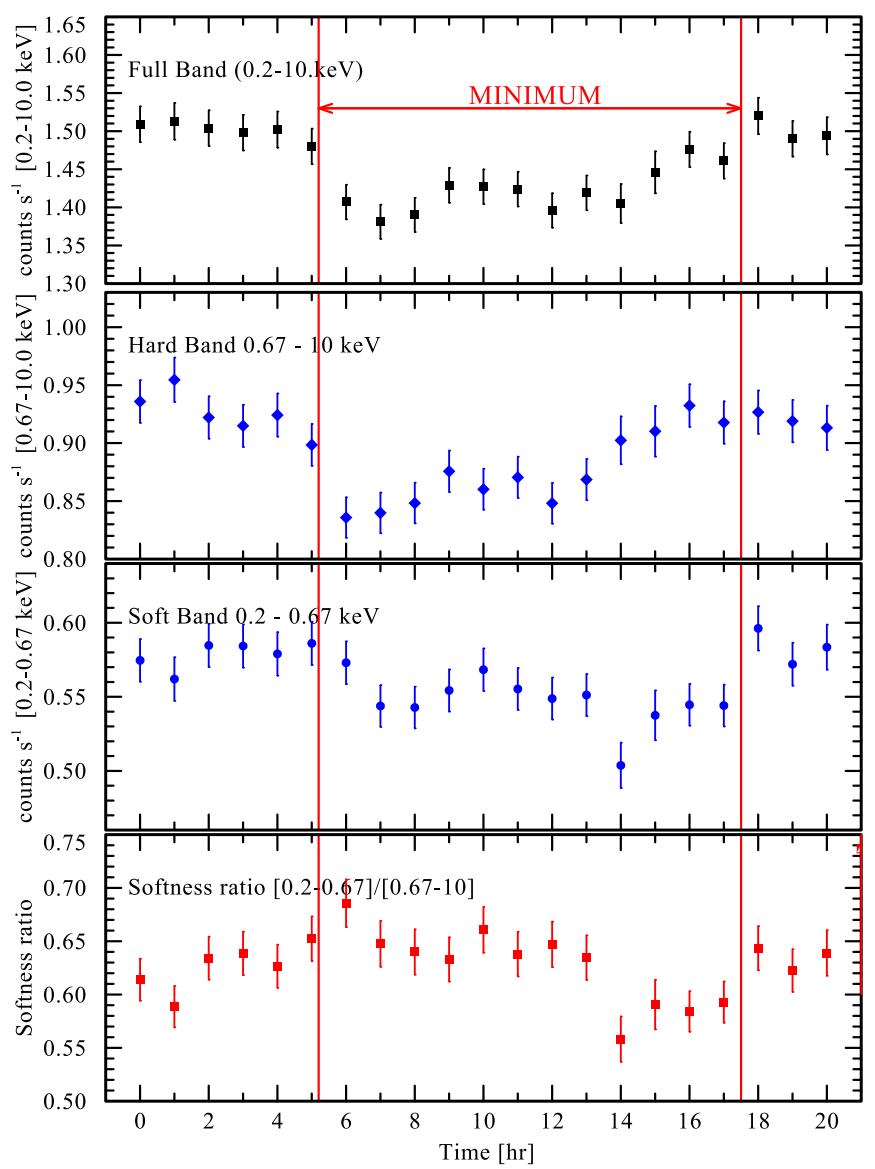

Figure 4. EPIC pn background-subtracted X-ray light curves of the first $\xi$ Per observation. The data were binned to $1 \mathrm{hr}(3.6 \mathrm{ks})$. The horizontal axis denotes the time after the beginning of the observation in hours. The vertical axes in the three upper panels show the count rate as measured by the EPIC pn camera The error bars $(1 \sigma)$ correspond to the combination of the error in the source counts and the background counts. The three upper panels show the light curves in different bands, as indicated. The bottom panel shows the "softness ratio" obtained by dividing the count rate in the $0.2-0.67 \mathrm{keV}$ band by the count rate in the $0.67-10.0 \mathrm{keV}$ band. The vertical red lines encompass the time interval we define as the X-ray minimum.

acquired. The optical monitor was not operating, due to the excessive optical brightness of $\xi$ Per. The data were reduced using the most recent calibrations and the Science Analysis System (SAS) v.15.0. The spectra and light curves were extracted using standard procedures. The background area was chosen to be nearby the star and free of X-ray sources.

$\mathrm{X}$-ray light curves were constructed from the EPIC data to examine the temporal variability. The EPIC data were binned into $1 \mathrm{hr}(3.6 \mathrm{ks})$ time bins and three broadband wavelength bins: the "full" $(0.2-10.0 \mathrm{keV})$ EPIC bandpass, the "soft" $(0.2-0.67 \mathrm{keV})$ band, and the "hard" $(0.67-10.0 \mathrm{keV})$ band. The X-ray light curves for these bands measured by the EPIC pn camera, as well as a "softness" ratio of the soft and hard bands, are shown in Figures 4 and 5 for the first and second observations, respectively. The light curves measured by the EPIC MOS cameras are similar. All of the X-ray light curves show statistically significant variability of $\gtrsim 10 \%$ on a timescale of hours. As pointed out in Section 1, similar variability has been detected in all other sufficiently well-studied O stars.

The full-band light curves in Figures 4 and 5 contain time intervals when the count rate is lower and higher than the average. We denote these time intervals as "minimum" and

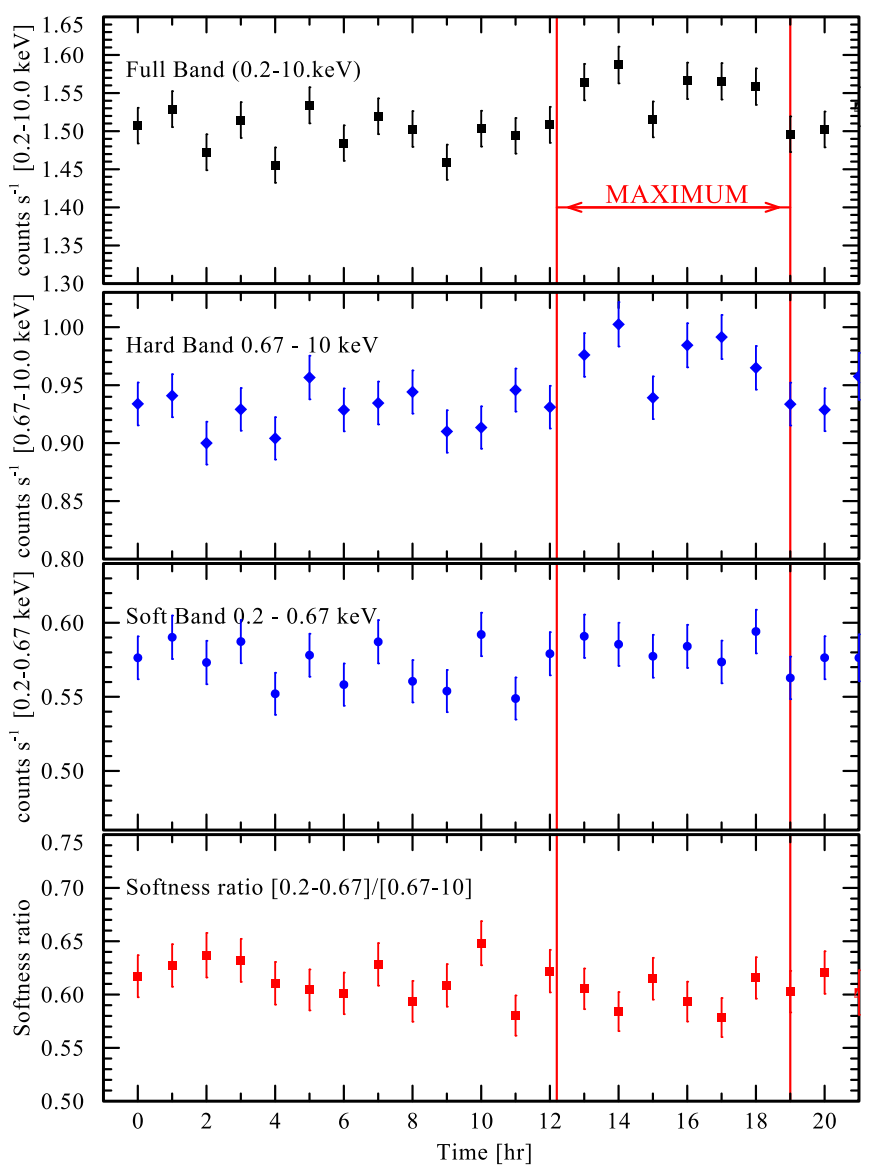

Figure 5. Same as Figure 4, but for the second observation. The vertical red lines encompass the time interval we define as maximum.

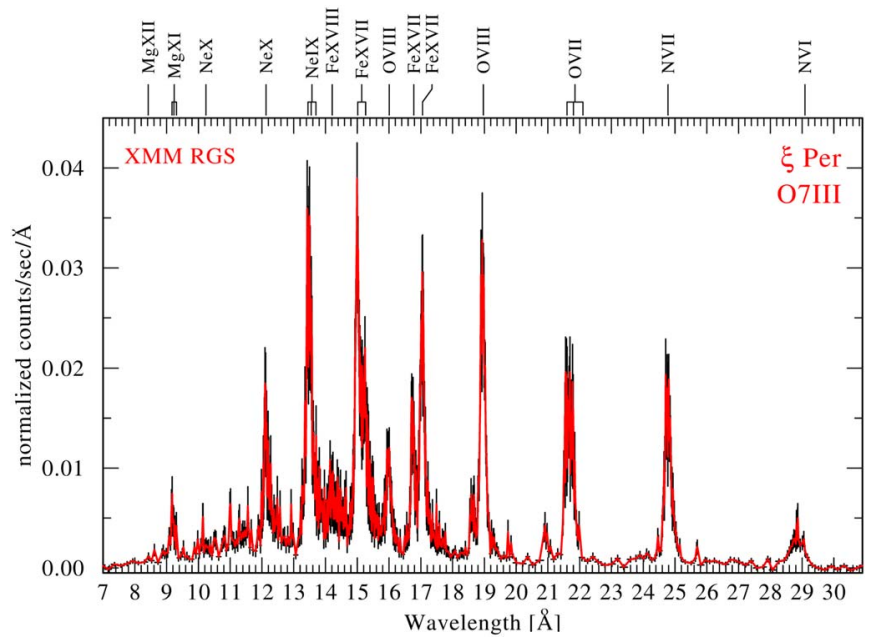

Figure 6. XMM-Newton combined RGS1 +2 spectrum of $\xi$ Per integrated over the full exposure time (red curve). Strong emission lines are identified. The error bars (black) correspond to $3 \sigma$.

"maximum." The minimum occurred during the first observation and the maximum during the second observation. The bottom panels in Figures 4 and 5 show the ratio of counts rates in soft and hard bands, termed the "softness ratio."

Finally, Figure 6 shows the merged RGS spectrum of $\xi$ Per. Overall, its mean spectrum is not unusual for an $\mathrm{O}$ star of its temperature (Waldron \& Cassinelli 2007; Walborn et al. 2009; Cohen et al. 2014). 


\section{Analysis}

\subsection{Variable UV Wind Lines}

Our ultimate goal is to find a link between the variability seen in the UV wind lines and the X-rays. This would imply that whatever causes the UV DACs is also responsible for the $\mathrm{X}$-ray variability. But first, we must demonstrate that the wind activity during our observations was similar to that expected from the earlier IUE time series. Because the new data are temporally scattered and sparsely sample the phase, we appeal to previous $I U E$ observations for guidance. Consequently, we begin the analysis with a reexamination of existing IUE observations. First, we explain why we elect to analyze specific lines over a limited range in velocity. Next, we introduce a simple model that captures the variations seen in the IUE data. We then use the model to demonstrate that the new STIS data are consistent with the previous observations and to determine the relative phases of the UV lines and the XMM-Newton light curve. Finally, we examine how the XMM-Newton spectra respond to changes in the $\mathrm{X}$-ray intensity.

The available UV spectral time series include two wind lines that are well developed but unsaturated, the property required to study variations. These are the Si IV $\lambda 1400$ resonance doublet and the N IV $\lambda 1718$ excited-state line. We would like to use these UV lines to extract information about the source of the DACs. This means that we want to sample the wind as close to the source as possible, at low wind velocity. At high velocity the modulation of the flux can become complex owing to the evolution of the structures responsible for the DACs as they move through the turbulent wind and to the overlap of different structures in velocity (e.g., Puls et al. 1993; Cranmer \& Owocki 1996).

The N IV $\lambda 1718$ excited-state line is of particular importance. The lower level of this line depends on the stellar radiation field to populate it (Olson 1981). Therefore, we can be certain that it samples the wind very close to the star (Massa \& Prinja 2015). Extracting meaningful information from the Si IV $\lambda 1400$ doublet at low velocity is problematic because the separation of the components is $w=0.8$, where $w=v / v_{\infty}$, $v=c\left(\lambda-\lambda_{0}\right) / \lambda$, and $\lambda_{0}$ is the rest wavelength of the line. This means that the high-speed wind absorption from the red component affects the absorption by the blue component between $-0.2 \leqslant w \leqslant 0.0$. As a result, for this line it is not possible to extract an uncontaminated measure of the wind activity close to the star. In contrast, all of the absorption by the red component is affected by emission from the blue component. However, it is well known that, for P Cygni lines, the emission originates from throughout the wind and tends to be far less variable than the blue absorption, which originates from a column between the observer and the stellar disk (e.g., Massa et al. 1995; Kaper et al. 1996). Consequently, one can expect the low-speed absorption of the $1402 \AA$ component to be relatively free of variable influences, due to the weak emission from the $1393 \AA$ component. Therefore, we include data from this component in our analysis.

To characterize the variations, we employ the well-studied IUE time series of $\xi$ Per obtained in 1994 October (e.g., de Jong et al. 2001; Massa \& Prinja 2015). We begin by normalizing the spectra over the range $1.0 \leqslant w \leqslant 1.5$. This accounts for uncertainties in the absolute flux levels of IUE high-dispersion spectra. Figure 7 shows the flux variation in N IV $\lambda 1718$ averaged over the region $-0.2 \leqslant w \leqslant 0.0$, where the fluxes are normalized by their mean value and plotted against phase, relative to the 2.086-day period determined by de Jong et al. Points from even and odd cycles are plotted with different symbols to examine whether the variations caused by the presumably two distinct spots differ. There does not appear to be a discernible difference. The flux in the line varies by about $10 \%$. The Si IV $\lambda 1402$ fluxes were similarly binned and normalized to obtain the light curve shown in Figure 7. It is clear that the shape and amplitude of the flux variations in N IV and Si IV are very similar.

Echelle data are subject to systematic errors that arise from placement of the object in the aperture and instantaneous telescope focus. Because these can be much larger than the statistical errors, we used a direct method to estimate the errors. First, a nearby continuum location was selected, and the same number of wavelength points used to bin the line data between $-0.2 \leqslant v / v_{\infty} \leqslant 0.0$ were binned in the normalized continuum region of each spectrum. Next, we calculated the sample variance of the binned points. The results were $\gtrsim 0.01$. Since the continuum points are divided by a larger mean flux than in the lines, the errors in the line data are expected to be of order 0.02 .

We would like to codify the shapes of the light curves shown in Figure 7 by a function that can faithfully represent them and also provide some insight into the structure of the wind. Such a function will also be useful for analyzing the sparsely sampled STIS data described below. To do this, we developed a simple model consisting of a wind whose strength differs from the global flow only in a pair of identical, uniform, equatorial, circular spots separated by $180^{\circ}$. Our simple model is for two diametrically opposed spots on the equator of the star. In fact, if $\sin i \sim 1$, these diametrically opposed spots could also be at higher latitude. Similarly, if the rotation period of $\xi$ Per is 2.068 days and $i \simeq 45^{\circ}$ (see the Appendix), the light curve could be due to a single, high-latitude spot. Regardless, in all cases, the major factors that determine the shapes of the model light curves are the size of the spots and the fraction of the cycle that they are occulted by the star. As we shall see, the simplest case of two equatorial spots provides an adequate fit to the data. Consequently, there is little reason for adding additional parameters to the model since they would be poorly determined. Nevertheless, we emphasize that the variability could be due to spots at higher latitude or even due to a single, high-latitude spot if the rotation period is 2.086 days.

Our two-spot model is described in the Appendix, where it is shown that it results in a normalized light curve, $r\left(\phi_{i}\right)$, of the form

$$
r\left(\phi_{i}\right)=\frac{1+C a\left(a_{0}, \phi_{i}+\phi_{0}\right)}{1+C\left\langle a\left(a_{0}, \phi+\phi_{0}\right)\right\rangle} .
$$

In this expression, $\phi_{i}$ is the phase of the $i$ th measurement relative to the 2.086-day period, $C$ is a constant, $a\left(a_{0}, \phi_{i}+\phi_{0}\right)$ is the fraction of the star covered at $\phi_{i}, a_{0}=\pi R_{\text {spot }}^{2} /\left(\pi R_{\star}^{2}\right)$ is the fractional area of the spot at $\phi=0$, and $\phi_{0}$ is the phase shift needed to align the model curve with the observations. The shape of $r(\phi)$ depends on the three parameters: $C, a_{0}$, and $\phi_{0}$. Some properties of the function are discussed in the Appendix. Fits to the $I U E$ data using this function are shown in Figure 7. The fits were determined by unweighted, nonlinear least squares, using the Interactive Data Language (IDL) procedure MPFIT developed by C. Markwardt. ${ }^{6}$ The parameters derived from the fits are listed in

\footnotetext{
6 The Markwardt IDL Library is available at http://cow.physics.wisc.edu/ $\sim$ craigm $/$ idl/.
} 

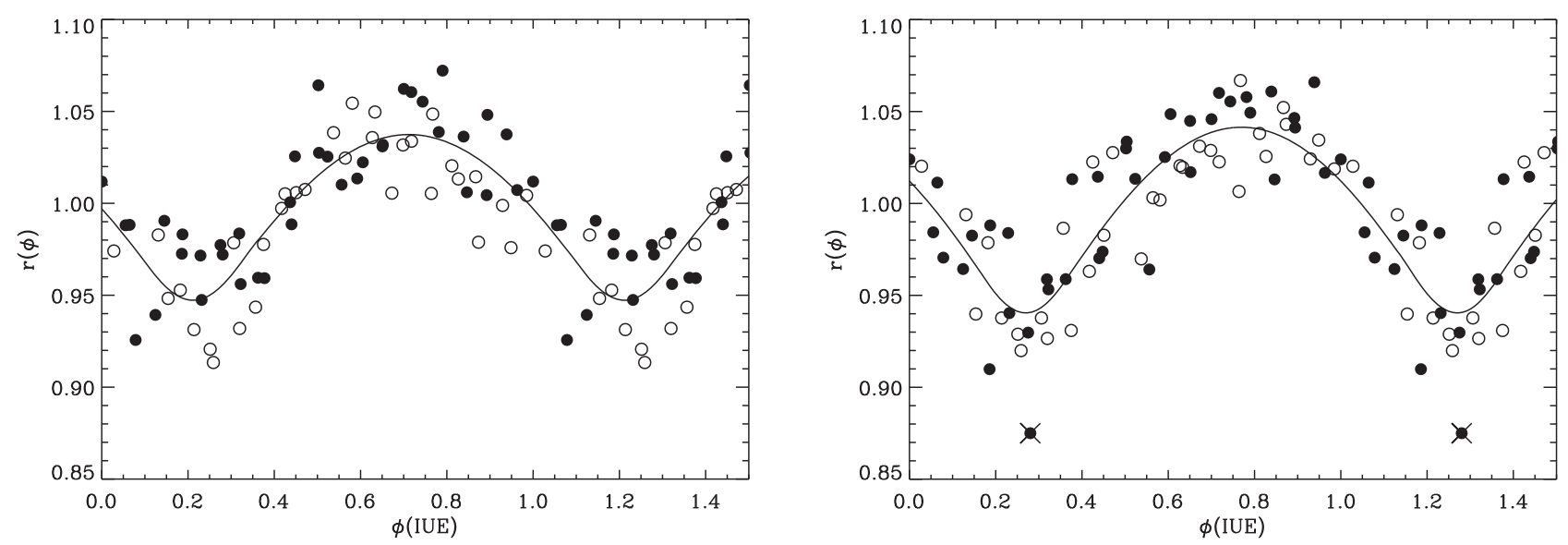

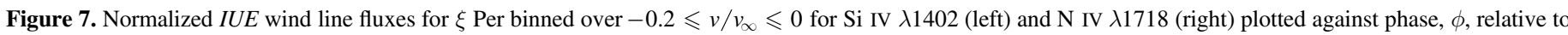

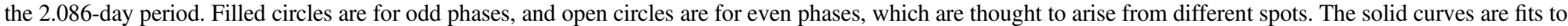

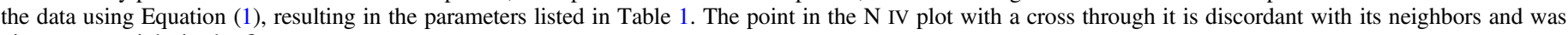
given zero weight in the fit.

Table 1, along with the rms residuals of the fits, which are comparable to the observational errors. Note that there is a significant phase difference between the Si IV and N IV light curves, $\phi_{0}(\mathrm{Si}$ IV $)-\phi_{0}(\mathrm{~N}$ IV $)=0.057 \pm 0.016$.

A caution is in order concerning the monovariate errors listed in Table 1. The error quoted for $\phi_{0}$ is quite robust, because the full error covariance matrix of the parameter errors shows that it is independent of the other variables, with correlation coefficients all less than 0.15 . In contrast, the parameters $C$ and $a_{0}$ are highly correlated, for reasons given in the Appendix. The correlation coefficients for these two parameters are -0.89 and -0.91 for the $\mathrm{N}$ IV and Si IV fits, respectively. This means that even though the derived values have small monovariate errors, their values can be changed substantially and have little effect on the quality of the fits, as long as $a_{0} C$ is held constant.

A set of STIS light curves were prepared in the same way as the IUE data. Figure 8 shows the STIS fluxes averaged over $-0.2 \leqslant w \leqslant 0.0$, for both N IV $\lambda 1718$ and Si IV $\lambda 1402$. Both curves are normalized by their mean. Observational errors were determined as before and are also expected to be about 0.02 in the lines.

Although the parameters $a_{0}$ and $C$ are highly correlated, the $I U E$ data are able to determine them reasonably well because the curves are so well sampled. This is not the case for the STIS data. To constrain the fits to the STIS data, $a_{0}$ was fixed at 0.1 , which is similar to the IUE values. This does not degrade the quality of the fits compared to those with both parameters free, and it does not alter the value of $\phi_{0}$. Its only effect is that the values of $C$ determined by the fits are closer to the $I U E$ results. Without the constraint, very different values of $a_{0}$ and $C$ result, but their product, $a_{0} C$, is nearly identical to that of the parameters listed in Table 1 . The important point is that all reasonable fits result in $a_{0} C>0$. As described in the Appendix, this implies that the spots are brighter than their surroundings.

The fits to the STIS data are displayed in Figure 8, and their parameters are listed in Table 1. Once again, the fits are considered excellent, with rms residuals of $\simeq 2 \%$. The relative phase between the N IV and Si IV fits determined by the STIS curves is $0.127 \pm 0.049$. As with the IUE data, the shift appears to be real. Although the STIS difference is larger than
Table 1

IUE Model Parameters

\begin{tabular}{lcccc}
\hline \hline Line & $\phi_{0}$ & $a_{0}$ & $C$ & rms \\
\hline IUE 1402 & $-0.288 \pm 0.012$ & $0.112 \pm 0.021$ & $1.02 \pm 0.20$ & 0.022 \\
IUE 1718 & $-0.231 \pm 0.011$ & $0.094 \pm 0.013$ & $1.34 \pm 0.21$ & 0.022 \\
STIS 1402 & $-0.531 \pm 0.025$ & $0.100 \pm 0.000$ & $1.17 \pm 0.40$ & 0.021 \\
STIS 1718 & $-0.414 \pm 0.042$ & $0.100 \pm 0.000$ & $0.97 \pm 0.37$ & 0.021 \\
XMM-Newton & $-0.335 \pm 0.020$ & $\cdots$ & $\cdots$ & $\cdots$ \\
\hline
\end{tabular}

$I U E$, the difference between the two is only slightly larger than $1 \sigma$, i.e., $0.070 \pm 0.052$, or $1.4 \sigma$. Thus, both the STIS and IUE data yield consistent amplitudes and relative phases. It is remarkable that data taken $21 \mathrm{yr}$ apart appear so similar.

The values in Table 1 show that $C$ is $\sim 1$ for the $I U E$ data. We employ Equation (5) in the Appendix to examine the implications of this value. First, consider the case where the bright spot results from a reduction in the photospheric absorption line in the area occupied by the spot. To obtain $C \simeq 1$ requires $f_{s} / f_{0} \simeq f_{e} / f_{0}+2$, or a weakening of the line flux by more than a factor of 2 . Next, consider the case where the bright spot is a region that is free of low-speed wind absorption and the surrounding wind has an optical depth of $\tau_{w}$. In this case, the same equation becomes $\left(f_{s}-f_{s} e^{-\tau_{w}}\right) /\left(f_{s} e^{-\tau_{w}}+f_{e}\right)$. If $\tau_{w} \simeq 1$, then for $\mathrm{C}=1, f_{e} / f_{s} \simeq 0.25$. Both results are reasonable.

There are a few properties of the fits worth emphasizing. First, the rms residuals are $\sim 2 \%$, which is similar to the expected errors. This is somewhat surprising, considering that the observations were obtained over several cycles and are possibly due to two distinct spots. Second, both sets of data imply that there is a significant phase difference between the $\mathrm{Si}$ IV and N IV light curves. Third, within the context of our simple model, the observed light curves can only be fit by curves with $C>0$, which implies that the spots are brighter than their surroundings (see the Appendix).

\subsection{Variable $X$-Ray Emission}

The XMM-Newton data were obtained over a 23-day interval (Figure 1) with a 21-day gap between two roughly day-long 

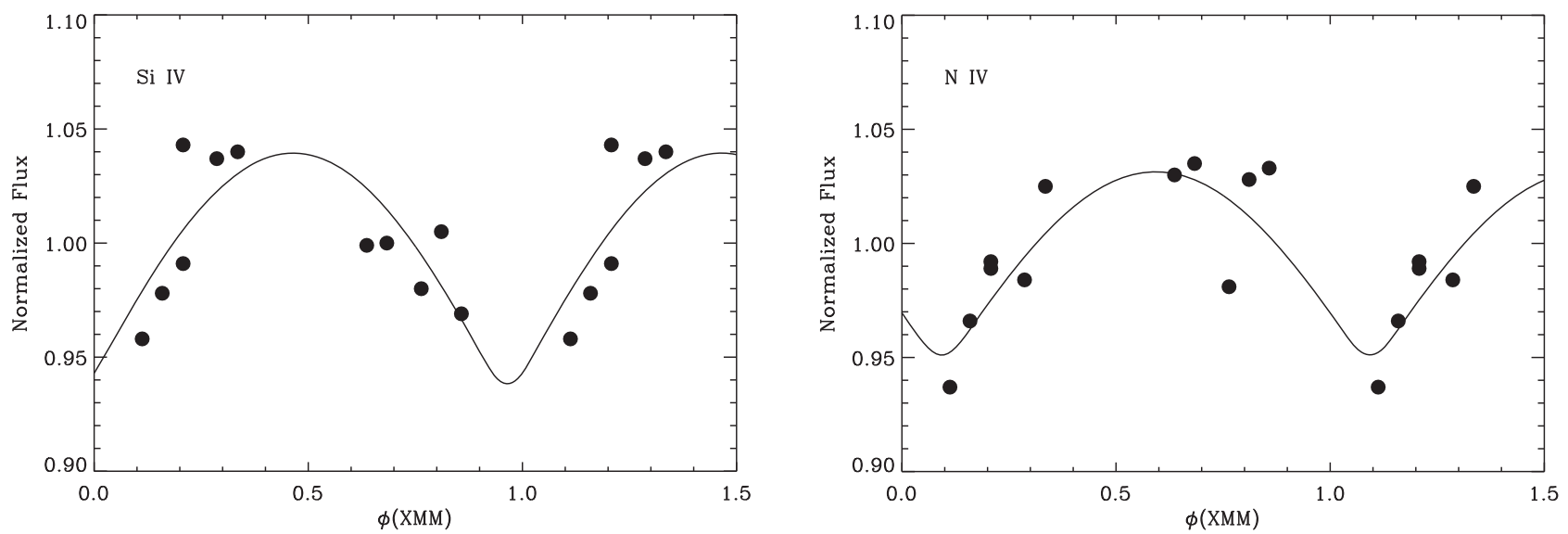

Figure 8. Model fit to the normalized STIS light curves for Si IV $\lambda 1402$ (left) and N IV $\lambda 1718$ (right). The parameters for the fits are given in Table 1 . The phases are relative to the first XMM-Newton observation.

exposures. Since the expected period is 2.086 days, the two observations are separated by about 10.5 cycles. This makes aligning the data very sensitive to the period assumed. Figure 9 shows the normalized XMM-Newton light curve, with different symbols indicating when the observations were obtained. The curve shape is distinctly different from those of the lines, with a broad shoulder after the minimum and a sharp peak before it. The amplitude of the variation is roughly $10 \%$. We also show a model light curve. Since the XMM-Newton light curve does not resemble those of the wind lines, we simply shifted the best model fit to the IUE N IV $\lambda 1718$ data until it agreed with the flux minima. Our best estimate for the phase shift and its error are listed in Table 1. The error was also estimated by emphasizing when the minimum was clearly not aligned with the model. It is unfortunate that the observations do not overlap in phase, although the two segments of the light curve do appear to join smoothly.

Figure 10 shows the STIS data shifted to align with the $X M M$-Newton fluxes to emphasize their mutual phase dependence. Using the $\phi_{0}$ values listed in Table 1, we determine the following sequence of events. First, the Si IV $\lambda 1402$ absorption begins to weaken (indicating a reduction in the Si IV column density). Next, the N IV $\lambda 1718$ absorption begins to weaken (indicating a reduction in the $\mathrm{N}$ IV column density). Finally, the $X M M$-Newton X-ray flux begins to increase.

To interpret these results as angular separations on the stellar surface, we must remember that the phases refer to the repeating DACs and that the stellar rotation period is probably twice as long. This means that the angular separations with respect to the star are half those inferred from the phase differences. Thus, the phase difference between Si IV and N IV translates to $10^{\circ} \pm 3^{\circ}$ (IUE) or $23^{\circ} \pm 9^{\circ}$ (STIS), and the phase difference between N IV (STIS) and the X-ray curve becomes $14^{\circ} \pm 8^{\circ}$.

Next, we examine how the spectral properties of the X-rays behave as a function of phase. Figure 11 shows the EPIC fluxes as a function of phase (normalized by their mean) and the softness ratio defined in Section 3.3 normalized by their mean. It is clear that the two quantities are anticorrelated, with the softness ratio decreasing when the $\mathrm{X}$-ray flux increases and vice versa, i.e., the spectrum becomes soft when the flux is weak and hard when the flux is strong. This is at odds with expectations. It has been proposed that $\mathrm{X}$-ray variability in $\mathrm{O}$ stars might result from additional absorption of X-rays by the

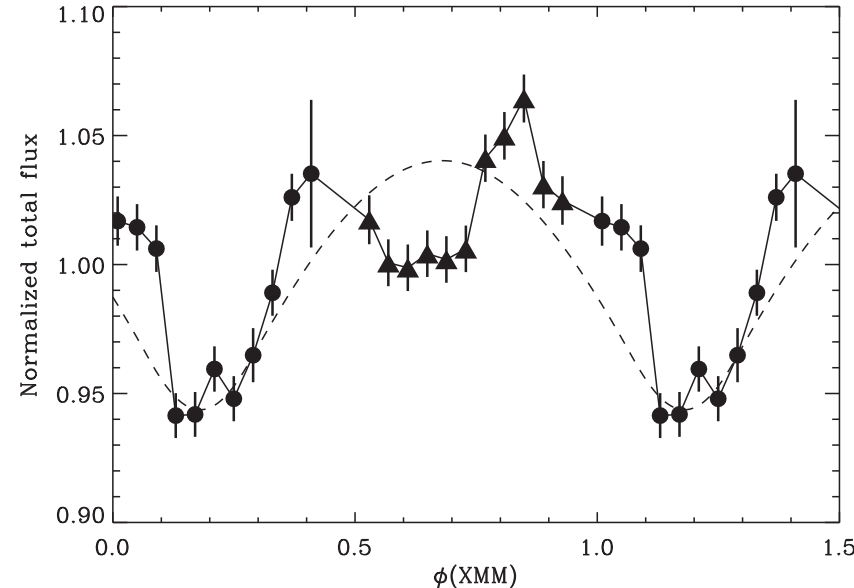

Figure 9. Normalized sum of the XMM-Newton pn, MOS1, and MOS2 data and their $1 \sigma$ error bars along with the IUE N IV model (dashed curve) shifted to align with the minima. The phases are relative to the first XMM-Newton observation. Circles and triangles represent data from the first and second sets of observations.

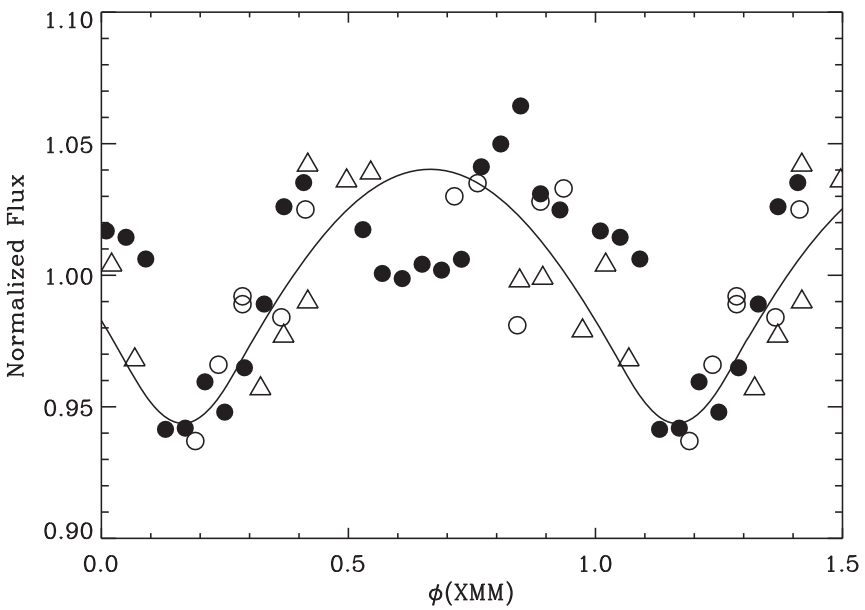

Figure 10. Normalized $X M M$-Newton and STIS data aligned. Filled circles are for the XMM-Newton fluxes, open circles for the N IV data, and open triangles for the Si IV data. The STIS data have been shifted to align them with the $X M M$-Newton data to emphasize how they all appear to follow a single period.

material in the CIRs (e.g., Oskinova et al. 2001). In this case, one would expect a harder and more strongly absorbed X-ray spectrum during the X-ray minimum, implying that the softness 


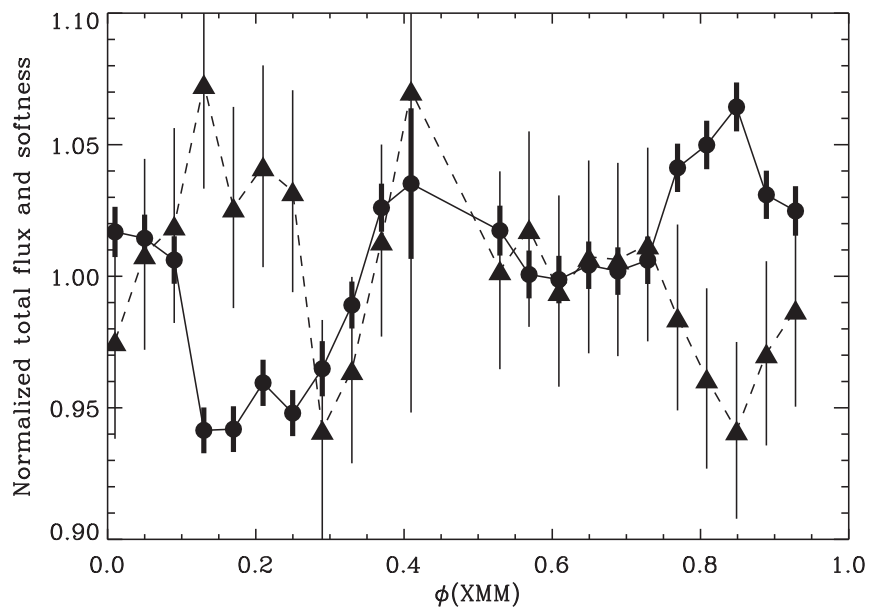

Figure 11. Total EPIC flux (circles and solid lines) and softness ratio (triangles and dashed lines) plotted against phase. Both curves are normalized by their means.

ratio should drop during the X-ray minimum. Instead, Figure 11 shows that the X-ray emission is softer at minimum than at maximum.

Finally, we examine the line profiles of the strongest lines in the RGS spectra. For an on-axis source, the accuracy of the RGS first-order wavelength scale is $0.005 \AA$. Figure 12 shows the profiles of N VII $\lambda 24.8$, O VIII $\lambda 18.97$, and Fe XVII $\lambda 17.1$, whose ionization ranges cover $552-667 \mathrm{eV}, 739-871$ $\mathrm{eV}$, and 490-1256 eV, respectively. Comparison of the smoothed profiles obtained at minimum and maximum reveals an important trend: the strong emission lines observed at maximum are more strongly blueshifted than at the minimum. However, this trend is not as evident in N VII Ly $\alpha$ (discussed below), which appears centered on its rest wavelength (taking into account line blending) at both minimum and maximum. Macfarlane et al. (1991) and Ignace (2001) showed that emission lines produced by optically thin radiation suffering $\mathrm{K}$-shell absorption in stellar winds are expected to be skewed and blueshifted. Moreover, the blueshift and the line shape are sensitive to wind absorption. The higher the stellar wind opacity is, the more blueshifted are the emerging emission lines. With the advent of highresolution X-ray spectroscopy, this formalism was extended to accelerating stellar winds (Owocki \& Cohen 2001; Waldron \& Cassinelli 2001) and clumped stellar winds (Feldmeier et al. 2003). Hence, the stronger blueshift of $\mathrm{X}$-ray emission lines during $\mathrm{X}$-ray maximum is possibly due to stronger wind opacity at maximum than at minimum, but the exact details depend on the geometry of the emitting plasma. Nevertheless, the fact that the N VII $\lambda 24.78$ line does not appear to shift is consistent with this explanation. This line is a blend, and its modeling is complicated, but the relative change seen in other lines is not present. One possible interpretation is that its line formation region is different from the heavier ions. For example, Waldron \& Cassinelli (2007) pointed out in their analysis of highresolution X-ray spectra of $\mathrm{O}$ stars that the line formation regions of heavier ions are closer to the stellar surface compared to the lines of lighter ions, again suggesting that the increase in X-ray flux is caused by a source deep in the wind.

\section{Summary and Discussion}

The observational results of this paper can be summarized as follows:

1. The IUE light curves of the low-velocity absorption in the UV wind lines of $\xi$ Per vary with a period of 2.086 days and amplitudes of $\sim 10 \%$.

2. The periods and shapes of the new STIS light curves are consistent with IUE light curves obtained $22 \mathrm{yr}$ earlier.

3 . Both the IUE and STIS light curves for Si IV $\lambda 1402$ and $\mathrm{N}$ IV $\lambda 1718$ are shifted in phase, with the N IV curve lagging the Si IV curve by a phase of $\sim 0.06$.

4. Variations in the X-ray flux are consistent with a 2.086day period and vary with an amplitude of $\sim 10 \%$ but have a distinctly different curve shape. Nevertheless, if its minimum is aligned with the minimum in the $\mathrm{N}$ IV line light curve, it is also shifted in phase, lagging the $\mathrm{N}$ IV curve by a phase of $\sim 0.08$.

5. The sequence of events is $\mathrm{Si}$ IV maximum, N IV maximum, and X-ray light maximum.

6. The X-ray flux is softer at minimum than at maximum, suggesting that the X-rays are more strongly absorbed at maximum.

7. The profiles of the X-ray lines appear to become more skewed toward high velocity at X-ray maximum. Furthermore, the lines of heavier ions are more skewed at maximum than those of lighter ions. These observations are consistent with the radiation from hotter plasma suffering higher absorption at maximum.

8. While the XMM-Newton observations cover the 2.086day period quite well, none of them sample the same phase more than once. As a result, we cannot firmly conclude that the X-ray light curve repeats.

If we interpret the observations in terms of our spot model, then we can infer the following:

1. Both the IUE and STIS light curves are well represented by a simple spot model with two identical, bright surface spots located on opposite sides of the star. Each spot covers roughly $10 \%$ of the projected stellar disk, implying radii $R_{\text {spot }} \sim 0.3 R_{\star}$ and an angular diameter of $\sim 37^{\circ}$. These are only representative values, as spots at different latitudes would have somewhat different areas.

2. By bright spots, we mean regions of reduced low-velocity absorption. This could indicate less photospheric absorption or a smaller Sobolev optical depth at low velocity. The latter could be due to a localized increase in the wind velocity gradient, reduction in the mass-loss rate, or a shift in the ionization state.

3. The similar flux variations in lines with very different ionization potentials, oscillator strengths, and formation properties suggest that their variations are due to variable covering factors and not to varying optical depths.

4. Assuming a rotation period of $2 \times 2.086$ days, the phase differences between events imply that the Si IV minimum occurs first, then the $\mathrm{N}$ IV minimum occurs $23^{\circ}$ after that, and the X-ray minimum occurs $14^{\circ}$ after that.

5. The X-ray spectrum at maximum is more absorbed than at minimum, which suggests that the source of the variable X-ray flux originates deep in the wind.

6. The X-ray line profiles are more skewed to high velocity at X-ray maximum. The interpretation of this result 

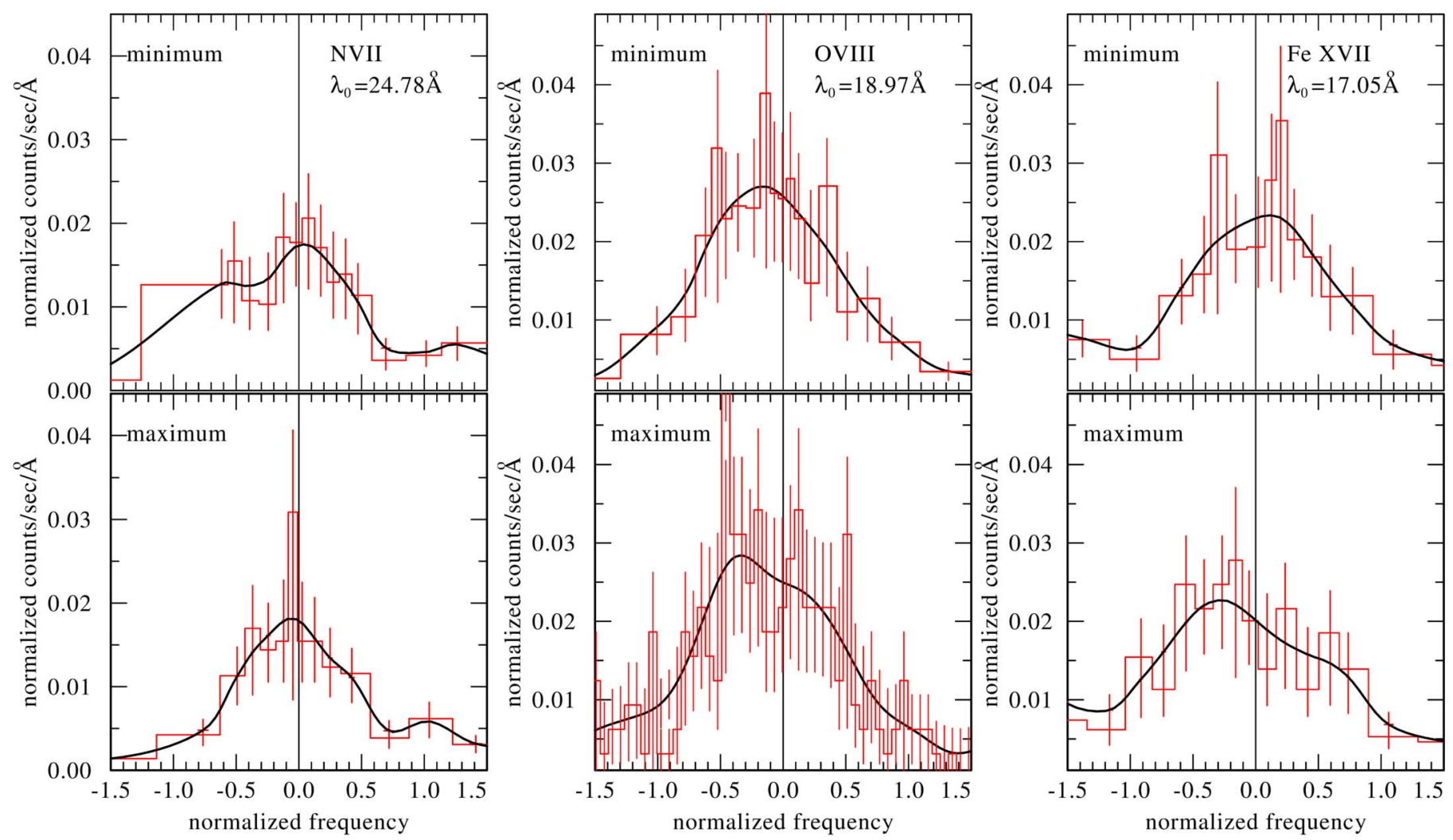

Figure 12. Mean profiles for RGS spectra obtained at X-ray minimum (top) and maximum (bottom) for N VII (left), O VIII (middle), and Fe XVII (right) plotted against $v / v_{\infty}$, where $v_{\infty}=2450 \mathrm{~km} \mathrm{~s}^{-1}$. These lines sample progressively higher ionized gas. The smooth curves are the data smoothed by a Gaussian with a full width of $0.08 \AA$, in order to accentuate the positions of the curve maxima.

depends on the geometry of the emitting and absorbing regions, making it strongly model dependent.

7. The fact that the low-velocity (near-surface) wind line variations correlate with the X-rays, together with the observation that the X-rays are more strongly absorbed at maximum, suggests that both variations occur very near the stellar surface.

In addition, we note that whatever the physical mechanism that causes the X-ray and UV wind line variations must also cause less than a few hundredths of a magnitude in the photometric variability at optical wavelengths (Ramiaramanantsoa et al. 2014). Furthermore, this mechanism must be quite common, since most hot stars with well-developed but unsaturated wind lines that have been observed long enough display the temporal signature of CIR-like structures. This includes Wolf-Rayet stars and the central stars of planetary nebula. Although bolstered by fewer examples (see Section 1), it is becoming clear that whenever an OB star with a strong wind is observed long enough in X-rays, variability on a timescale of order the rotation period is also revealed.

Finally, we note that the persistence of two identical, diametrically opposed starspots for decades suggests an oblique magnetic rotator, where the spots are the magnetic poles. However, strong limits on the existence of even relatively weak dipole fields in $\xi$ Per and similar stars (David-Uraz et al. 2014) appear to make this mechanism doubtful.

In closing, we point out that a major caveat in our results is that we do not have direct evidence that the X-ray light curve repeats. Although our data cover a complete cycle and appear to splice together well, we lack observations for the same phase obtained during different cycles. Such observations would conclusively prove the connection between the UV wind lines and X-rays. Furthermore, if the two-spot model is correct, the two segments of our X-ray curve sample the same spot, and information on the diametrically opposed spot is totally lacking. Clearly, more X-ray data are needed. The observations that would cement the connection are repeated X-ray observations sampling the same phases of the light curve over several periods to determine whether it repeats. Nevertheless, the results of our new multiwavelength coordinated observing campaign imply that the X-ray variability in $\xi$ Per is correlated with the UV wind line variability. This suggests that the origin of the X-rays in this star, and possibly other single $\mathrm{O}$ stars, is linked to the same physical mechanisms responsible for the CIRs in stellar winds, which manifest themselves as periodic UV wind line variability.

D.M. and R.I. acknowlege support from grant No. HST-GO14180 provided by NASA through a grant from the Space Telescope Science Institute, which is operated by the Association of Universities for Research in Astronomy, Inc., under NASA contract NAS5-26555. L.O. is supported by the Deutsches Zentrum für Luft und Raumfahrt (DLR) grants FKZ 15 and OR 1809, and partially by the Russian Government Program of Competitive Growth of Kazan Federal University. We also thank the referee, whose comments greatly enhanced the clarity and conciseness of our presentation. 


\section{Appendix}

This appendix develops a simple model for the light variations we observe at low velocity in wind lines and examines some of its properties. We should point out that because we isolate a portion of the profile defined by velocity limits, the rotational velocity of the star will cause the geometric boundaries of the region to vary as the spot moves around the star. However, this effect is strongest as the spot clears the limb, when the projected area of the spot is smallest. In contrast, the most distinctive aspects of the light curves are determined from the spot crossing the face of the star, when the projected area is largest and the projected rotational velocity is smallest. For similar reasons, limb darkening is also ignored.

In modeling the light curves, we must first decide whether the 2.086-day period seen in the DACs is the rotational period and the observed variability is due to a single spot, or if the rotation period is $2 \times 2.086$ days and the variability is due to two nearly identical spots $180^{\circ}$ apart. The observed rotational velocity of $\xi \operatorname{Per}$ is $v_{\mathrm{obs}}=v_{\mathrm{eq}} \sin i=204 \mathrm{~km} \mathrm{~s}^{-1}$. Its rotation period, $P$, is probably one or two times the DAC period, $P_{\text {obs }}$, i.e., $P=P_{\text {obs }} n=2.086 n$ days, where $n$ is 1 or 2 . This consideration, along with estimates of the probable rotation velocity and stellar radius, places limits on $i$. First, we assume $v_{\text {eq }} \lesssim 300 \mathrm{~km} \mathrm{~s}^{-1}$; otherwise, the wind line spectrum of $\xi$ Per would probably be abnormal for its spectral type (e.g., Massa 1995; Prinja et al. 1997). This gives $204 / 300 \leqslant \sin i \leqslant 1$, or $43^{\circ} \leqslant i \leqslant 90^{\circ}$. Limits on the stellar radius restrict $i$ even further since $R_{\star}=$ $v_{\text {eq }} P /(2 \pi)=v_{\text {obs }} P_{\text {obs }} n /(2 \pi \sin i)=8.45 n / \sin i$. The Weidner \& Vink (2010) tables give a radius of $\sim 14.3$ for an O7III star. However, if $\xi$ Per is a little less luminous (class III/V), its radius could be as small as $R_{\min } \simeq 11.8$. If it is a bit more luminous (class I/III), its radius could be as large as $R_{\max } \simeq 17.9$. These imply $8.45 n / R_{\max } \leqslant \sin i \leqslant 8.45 n / R_{\min }$. Inserting the limiting radii gives $0.47 n \leqslant \sin i \leqslant 0.72 n$, or $28^{\circ} \leqslant i \leqslant 46^{\circ}$ for $n=1$ and $71^{\circ} \leqslant i \leqslant 90^{\circ}$ for $n=2$, where the $90^{\circ}$ limit for $n=2$ implies $R_{\min }=16.9$. The $v \sin i$ constraint restricts $i$ to $43^{\circ} \leqslant$ $i \leqslant 46^{\circ}$ for $n=1$, where the lower limit infers an $R_{\max }=12.4$. Together, the constraints give the following relations: $43^{\circ} \leqslant$ $i \leqslant 46^{\circ}$ and $11.8 \leqslant R_{\star} \leqslant 12.4$ for $n=1$, and $71^{\circ} \leqslant i \leqslant 90^{\circ}$ and $16.9 \leqslant R_{\star} \leqslant 17.9$ for $n=2$. Both situations are possible. The inclination, spot size, latitude, and intensity of the spots are all free parameters, although $i$ is rather strongly constrained for each case.

In the following, we adopt a two-spot model with equatorial spots for the simple reason that it is the easiest to calculate and adequately describes the light curves. However, one should keep in mind that opposing spots at higher latitudes are possible, as are one-spot models (if $P=2.086$ days) with the spot at high latitude. ${ }^{7}$

Our model consists of two identical, circular, equatorial spots separated by $180^{\circ}$. These spots can appear either brighter or darker than their surroundings, where these terms are referring to wavelengths very near the cores of strong lines. Therefore, a bright spot could be due to a region where the strength of the photospheric line is weakened or a region with weak low-velocity wind absorption. Similarly, a dark spot could be due to a region where the photospheric line has

\footnotetext{
7 Recently, Gordon et al. (2018) have derived a radius for $\xi$ Per of $\sim 11$, which favors one-spot models. However, unlike the other stars in their sample, the distance used to determine the radius was not from Gaia data, but from older, marginal Hipparcos data and indirect methods.
}

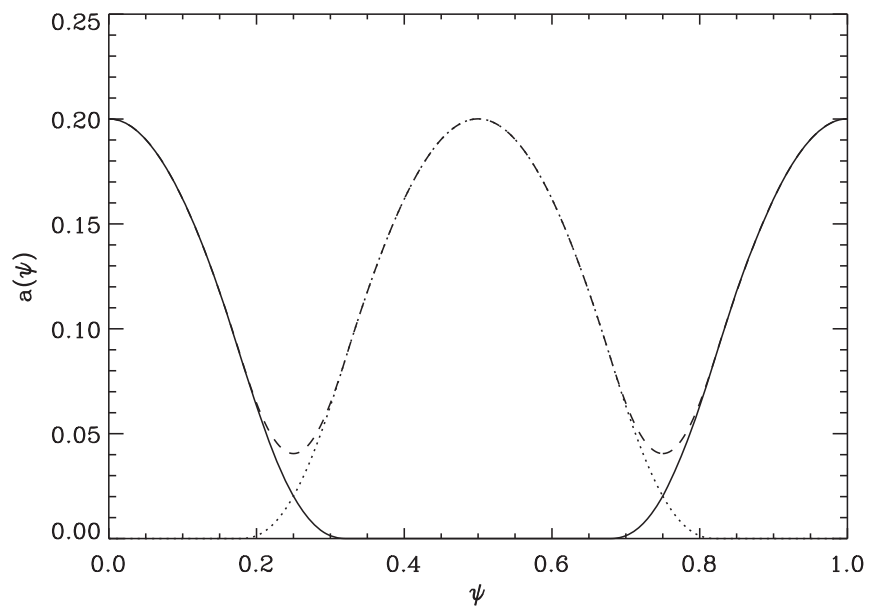

Figure 13. The solid curve is the projected area of a spot whose area is $0.2 \times \pi R_{\star}^{2}$ vs. phase, $\psi$, where $\psi=0$ occurs when the spot is centered along the line of sight. The dotted curve is the same curve displaced by 0.5 in $\psi$ and represents the projected area of a second spot $180^{\circ}$ away from the first spot. The dashed curve is the combination of the two and represents the projected area for the two spots.

strengthened or a region with strong low-velocity wind absorption.

To quantify the model, let the stellar flux be $f_{0}$, the flux from the spot be $f_{s}$, and the fraction of the star covered by the spot at rotational phase $\psi$ be $a(\psi)$. There is also a nonvariable contribution to the flux that comes from the light scattered throughout the wind, $f_{e}$. With these definitions, the flux varies as

$$
\begin{gathered}
f(\psi)=[1-a(\psi)] f_{0}+f_{s} a(\psi)+f_{e} \\
=\left(f_{0}+f_{e}\right)+\left(f_{s}-f_{0}\right) a(\psi) .
\end{gathered}
$$

The size of the spot is defined by the fraction of the disk covered when the spot is centered along the line of sight, $a_{0} \equiv a(0)$. In this case, the radius of the spot, $R_{s}$, is $R_{s}=\sqrt{a_{0}} R_{\star}$, where $R_{\star}$ is the stellar radius. Further, the angular size of the spot relative to the center of the star is $\theta_{0}=2 \sin ^{-1} \sqrt{a_{0}}$.

To examine the variations of a light curve sampled at $i=1, \cdots$, $N$ phases and normalized by its mean, $r\left(\psi_{i}\right)=f\left(\psi_{i}\right) /\langle f(\psi)\rangle$, we use a normalized version of the last equation, which is

$$
r\left(\psi_{i}\right)=\frac{1+C a\left(\psi_{i}\right)}{1+C\langle a(\psi)\rangle}
$$

where

$$
C=\frac{f_{s}-f_{0}}{f_{0}+f_{e}}
$$

is independent of phase.

The variable component of Equation (4) is the projected area of the spot. The solid curve in Figure 13 shows how the projected area of a circular, equatorial spot varies as a function of phase for a spot with $a_{0}=0.2$. At $\psi=0$, the spot is centered along the line of sight. As the star rotates, the area decreases owing to projection effects until it begins to be occulted by the star. Once totally eclipsed, the spot area remains zero until it begins to emerge from behind the star. The projected area then continues to increase until the spot returns to the center of the disk. The dotted curve is the solid curve 

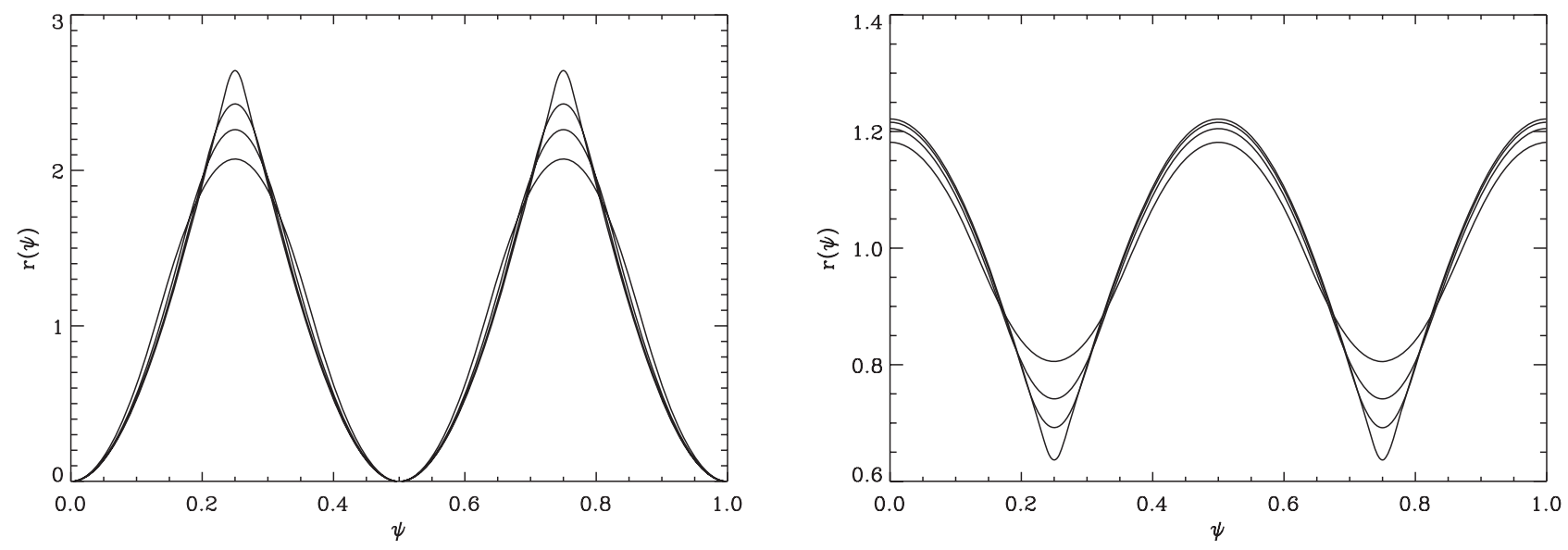

Figure 14. Left: model light curves for spots that are optically thicker than their surroundings plotted against rotation phase, $\psi$. The curves are for $a_{0}=0.50,0.25$, 0.10 , and 0.01 (bottom to top) and were calculated with $|C|=1 / a_{0}$ to make them similar over most of their range. Right: similar curves for the case where the spot is optically thinner than its surroundings. In this case curves with smaller $a_{0}$ have the deepest minima.

displaced in phase by 0.5 . When the two are added, the result is the projected area for two identical spots on opposite sides of the star. The combined curve is shown as the dashed curve. The important feature of this curve is that the maxima are broader than the minima. This is simply because the entire spot is behind the star for an interval of $\pi-\theta_{0}$, and some part of the spot is visible for an interval of $\pi+\theta_{0}$. Note that $\theta_{0}$ can be quite large. A spot with $a_{0}=0.1$ has an $R_{s}=0.32 R_{\star}$ and a $\theta_{0}=37^{\circ}$.

Equation (4) shows how the nature of the variability is determined by the quantity $f_{s}-f_{0}$ in the numerator of $C$. If $f_{s}>f_{0}$ (a bright spot), then $C>0$ and the line varies as $1+|C| a(\psi)$. If $C<0$ (a dark spot), the line varies as $1-|C| a(\psi)$. Figure 14 shows how the two families of $r(\psi)$ curves respond to spot size. In these figures, we set $C=1 / a_{0}$, which effectively normalizes the curves and emphasizes their differences. The curves are similar near the broad maxima (bright spots) or minima (dark spots), since the major factor dictating the projected area is the change in the inclination angle to the line of sight, which is independent of spot size. The curves differ near the minima (bright spots) or maxima (dark spots), which are shaped by how large the spot is and, therefore, how long it is partially visible.

It is important to note that because curves with $C=1 / a_{0}$ appear so similar (Figure 14), it will be difficult to extract independent values of $C$ and $a_{0}$ from the observations, especially if the region of the sharp extrema is not well sampled. This means that errors in the two parameters will be strongly correlated. As a result, changing the values of $C$ and $a_{0}$ so that $a_{0} C$ is constant will have little effect on the quality of the fits.

In the text, the fluxes are plotted against phase with respect to the 2.086-day period derived by de Jong et al. (2001). Following de Jong et al., we assume that the observed period of the flux variation is half of the rotation period, so we introduce $\phi=2 \psi$, where $\phi$ is the phase of the observations relative to the 2.086-day period. In this case, Equation (4) suggests fitting the observed variations with a model of the form

$$
r\left(\phi_{i}\right)=\frac{1+C a\left(a_{0} ; \phi_{i}+\phi_{0}\right)}{1+C\left\langle a\left(a_{0} ; \phi+\phi_{0}\right)\right\rangle},
$$

where $C$ and $a_{0}$ are the same as above and $\phi_{0}$ is the phase shift required to align the spot model with the observations. This is Equation (1) in the text.

\section{ORCID iDs}

Derck Massa (1) https://orcid.org/0000-0002-9139-2964 Richard Ignace iㅏ https://orcid.org/0000-0002-7204-5502

\section{References}

Castor, J. I., Abbott, D. C., \& Klein, R. I. 1975, ApJ, 195, 157

Cohen, D. H., Wollman, E. E., Leutenegger, M. A., et al. 2014, MNRAS, 439, 908

Cranmer, S. R., \& Owocki, S. P. 1996, ApJ, 462, 469

David-Uraz, A., Wade, G. A., Petit, V., et al. 2014, MNRAS, 444, 429

de Jong, J. A., Henrichs, H. F., Kaper, L., et al. 2001, A\&A, 368, 601

den Herder, J. W., Brinkman, A. C., Kahn, S. M., et al. 2001, A\&A, 365, L7

Feldmeier, A., Oskinova, L., \& Hamann, W.-R. 2003, A\&A, 403, 217

Feldmeier, A., Puls, J., \& Pauldrach, A. W. A. 1997, A\&A, 322, 878

Fullerton, A. W., Massa, D., \& Prinja, R. K. 2006, ApJ, 637, 1025

Fullerton, A. W., Massa, D., Prinja, R. K., Owocki, S. P., \& Cranmer, S. R. 1997, A\&A, 327, 699

Gordon, K. D., Gies, D. R., Schaefer, G. H., et al. 2018, ApJ, 869, 37

Hervé, A., Rauw, G., \& Nazé, Y. 2013, A\&A, 551, 83

Ignace, R. 2001, ApJL, 549, L119

Ignace, R., Gayley, K., Hamann, W.-R., et al. 2013, ApJ, 775, 29

Kaper, L., Henrichs, H. F., Nichols, J. S., et al. 1996, A\&AS, 116, 257

Kaper, L., Henrichs, H. F., Nichols, J. S., \& Telting, J. H. 1999, A\&A, 344, 231

Lobel, A., \& Blomme, R. 2008, ApJ, 678, 408

Lucy, L. B., \& White, R. L. 1980, ApJ, 241, 300

Macfarlane, J. J., Cassinelli, J. P., Welsh, B. Y., et al. 1991, ApJ, 380, 564

Massa, D. 1995, ApJ, 438, 376

Massa, D., Fullerton, A. W., Hutchings, J. B., et al. 2000, ApJ, 538L, 47

Massa, D., Fullerton, A. W., Nichols, J. S., et al. 1995, ApJ, 452L, 53

Massa, D., Fullerton, A. W., Sonneborn, G., \& Hutchings, J. B. 2003, ApJ, 586, 996

Massa, D., Oskinova, L., Fullerton, A. W., et al. 2014, MNRAS, 441, 2173

Massa, D., \& Prinja, R. K. 2015, ApJ, 809, 12

Mullan, D. J. 1984, ApJ, 283, 303

Nazé, Y., Oskinova, L. M., \& Gosset, E. 2013, ApJ, 763, 143

Nazé, Y., Ramiaramanantsoa, T., Stevens, I. R., Howarth, I. D., \& Moffat, A. F. J. 2018, A\&A, 609A, 81

Nichols, J., Huenemoerder, D. P., Corcoran, M. F., et al. 2015, ApJ, 809, 133

Olson, G. L. 1981, ApJ, 245, 1054

Oskinova, L. M., Clarke, D., \& Pollock, A. M. T. 2001, A\&A, 378, L21

Oskinova, L. M., Feldmeier, A., \& Hamann, W.-R. 2006, MNRAS, 372, 313

Oskinova, L. M., Hamann, W.-R., \& Feldmeier, A. 2007, A\&A, 476, 1331

Owocki, S. P., Castor, J. I., \& Rybicki, G. B. 1988, ApJ, 335, 914

Owocki, S. P., \& Cohen, D. H. 2001, ApJ, 559, 1108 
Prinja, R. K., \& Massa, D. 2010, A\&A, 521L, 55

Prinja, R. K., Massa, D., \& Cantiello, M. 2012, ApJ, 759L, 28

Prinja, R. K., Massa, D., \& Fullerton, A. W. 2002, A\&A, 388, 587

Prinja, R. K., Massa, D., Fullerton, A. W., Howarth, I. D., \& Pontefract, M. 1997, A\&A, 318, 157

Proffitt, C. R., TalaWanda Monroe, T., \& Dressel, L. 2017, Instrument Science Report STIS 2017-01(v1) (Baltimore, MD: Space Telescope Science Institute)

Puls, J., Markova, N., Scuderi, S., et al. 2006, A\&A, 454, 625

Puls, J., Owocki, S., \& Fullerton, A. 1993, A\&A, 279, 457

Ramiaramanantsoa, T., Moffat, A. F. J., Chené, A.-N., et al. 2014, MNRAS, 441, 910

Rauw, G., Hervé, A., Nazé, Y., et al. 2015, A\&A, 580A, 59
Steinberg, E., \& Metzger, B. D. 2018, MNRAS, 479, 687

Strüder, L., Briel, U., Dennerl, K., et al. 2001, A\&A, 365, L18

Sundqvist, J. O., Owocki, S. P., \& Puls, J. 2018, A\&A, 611A, 17

Sundqvist, J. O., Puls, J., Feldmeier, A., \& Owocki, S. P. 2011, A\&A, 528A, 64

Šurlan, B., Hamann, W.-R., Kubát, J., Oskinova, L. M., \& Feldmeier, A. 2012, A\&A, 541A, 37

Turner, M. J. L., Abbey, A., Arnaud, M., et al. 2001, A\&A, 365, L27

Walborn, N. R., Nichols, J. S., \& Waldron, W. L. 2009, ApJ, 703, 633

Waldron, W. L., \& Cassinelli, J. P. 2001, ApJL, 548, L45

Waldron, W. L., \& Cassinelli, J. P. 2007, ApJ, 668, 456

Weidner, C., \& Vink, J. S. 2010, A\&A, 524, A98

Wilms, J., Allen, A., \& McCray, R. 2000, ApJ, 542, 914 